\title{
Mapping of Weak Cathodoluminescence Signals
}

\author{
Nick C. Wilson ${ }^{1}$, Colin M. MacRae ${ }^{1}$ and Aaron Torpy ${ }^{1}$ \\ ${ }^{1}$ CSIRO Process Science and Engineering, Box 312, Clayton, VIC, 3169. \\ Corresponding Author's e-mail address: nick.wilson@csiro.au
}

Mapping of cathodoluminescent active materials to show growth zoning, trace element variations and valence is becoming more common [1]. The desire to capture cathodoluminescence (CL) maps from samples that are weakly emitting arises for two reasons. Firstly, the chemical spatial resolution in maps is related to the accelerating voltage used, and the drive for higher resolution $\mathrm{x}$-ray maps is leading to mapping being performed at lower voltages. Lowering the accelerating voltage to improve spatial resolution has the side effect of decreasing CL yield since the CL intensity from a given sample decreases as the accelerating voltage is lowered (Fig. 1a). The second reason to measure weak CL signals is in the examination of samples that are intrinsically or extrinsically weak CL emitters (Fig. 1b) and to date have been largely ignored by CL studies, for example band-gap measurements in hematite (Fig 2.) or to reveal subtle changes in CL intensity, such as radiation damage in quartz (Fig 3).

Generally hyperspectral CL mapping uses a ruled grating to spectrally disperse light across a charge coupled device (CCD) [2]. Whilst CCDs are relatively sensitive to light, they suffer from temperature effects in the form of dark noise and intensity variation with small changes in temperature. In order to map weakly emitting samples these effects must be minimized by a combination of hardware and software corrections. To retrieve the optical spectrum emitted from the sample, a background CCD dark noise spectrum is measured with the beam blanked, which is then subtracted from the measured spectrum. The shape of the background spectrum is caused by variations in the characteristics between individual CCD elements and incomplete charge clearance across the CCD, but this shape is obscured by readout and dark noise effects. Dark noise can be reduced by cooling of the CCD and for the short sampling times used in mapping this largely removes is contribution, while averaging many measurements of the background greatly reduces the readout noise. A remaining problem is subtle background shifts due to small temperature changes. Figure 3 shows the temperature drift of a Peltier cooled spectrometer across a map, with the peak-to-peak variation here equating to approximately 0.1 counts per pixel with a period of approximately 6 minutes. The periodicity is due to fluctuations in the ambient temperature. One method to correct for temperature drifts is to monitor the background in a set of blanked off CCD channels that are not exposed to light during acquisition. The signal from these blanked channels can then be used as a reference to shift the background spectrum up and down. A further reduction in the noise associated with the background signal of the blanked channels can be made by temporal filtering to remove the high frequency components. Fig 3a shows the raw signal from the blanked CCD pixels, which are then Fast Fourier Transformed (FFT) to filter the high frequency noise. The fitted dark noise map is then subtracted from the signal map, (Fig. 3b) pixel-by-pixel. The result of such a procedure allows subtle growth zones and structural modifications to be imaged.

\section{References:}

[1] C.M. MacRae et al., Microsc. Microanal. 18, (2013), p.1239-1245.

[2] C.M. MacRae et al, Microscopy Research and Technique, (2005), 67, p. 271-277. 

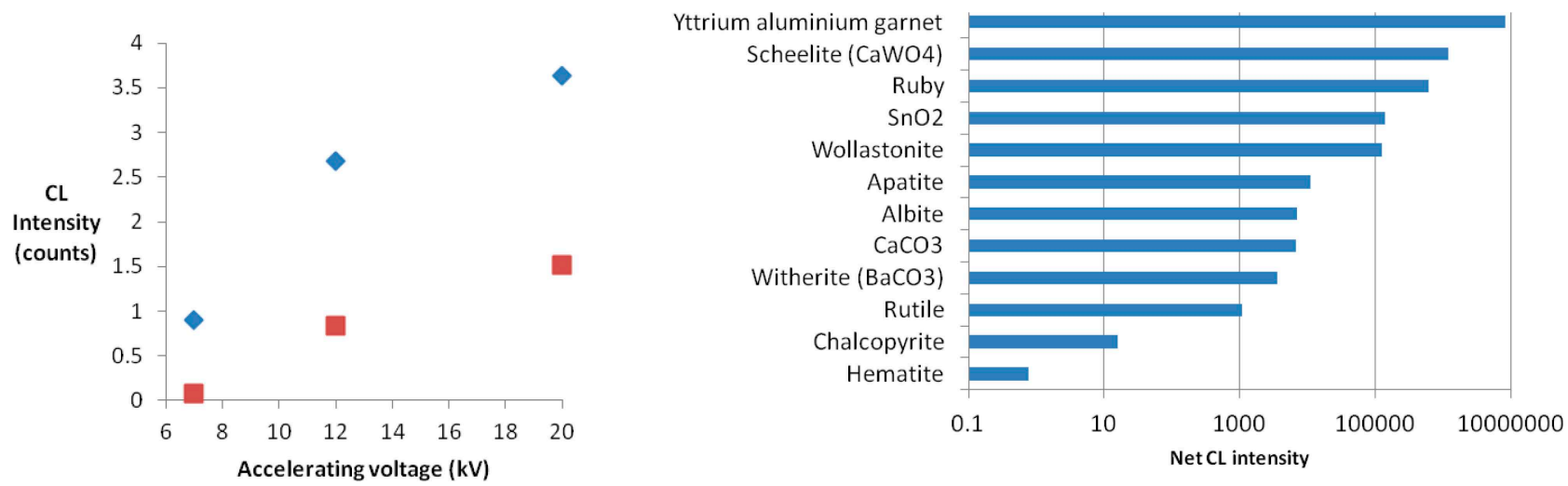

Figure 1. (a) CL intensity versus accelerating voltage on dolomite (red) and quartz (blue), (b) Net CL intensity at $25 \mathrm{kV}$ from a range of materials, showing the large dynamic range.

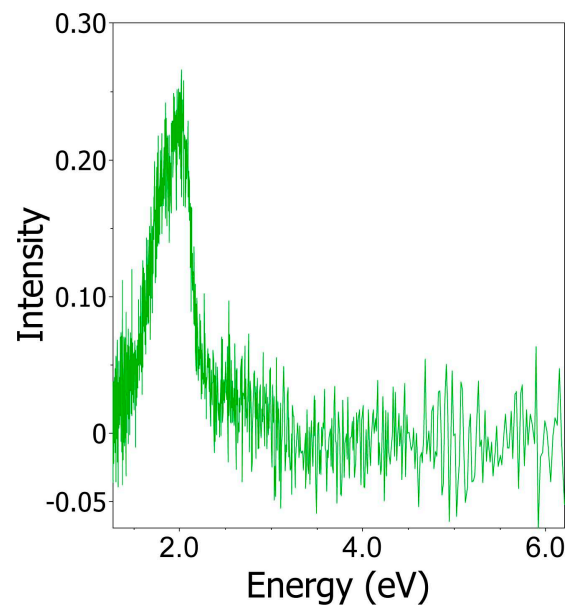

Figure 2. CL spectrum extracted from a from a hematite sample mapped at $25 \mathrm{kV}$ and $30 \mathrm{nA}$.
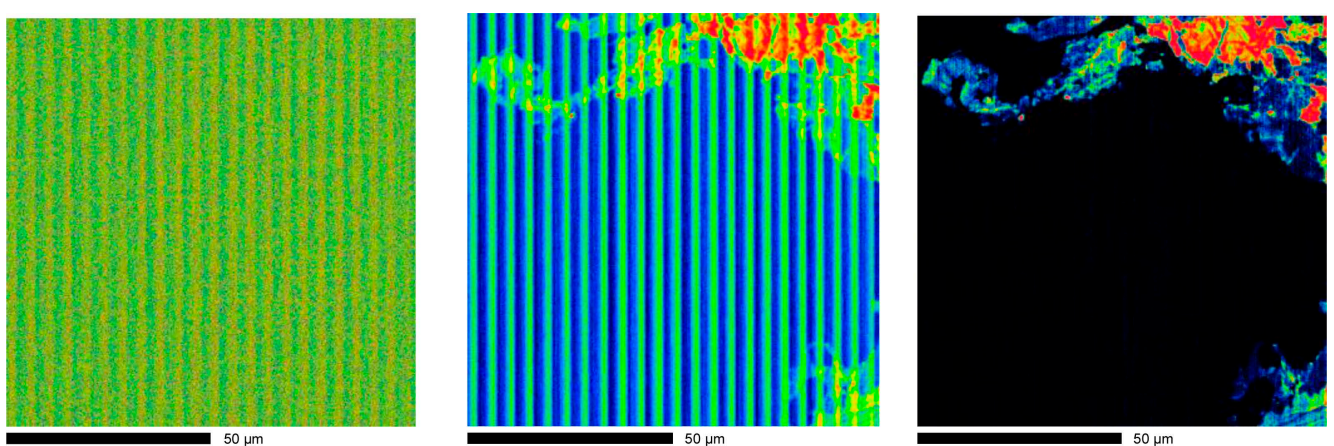

Figure 3. (a) Map of blanked off CCD pixels showing periodic noise due to temperature fluctuations. (b) Map collected on quartz showing significant dark noise oscillations. (c) Map of dolomite after the removal of dark noise drift. Note that the y-axis was the fast scan direction. 